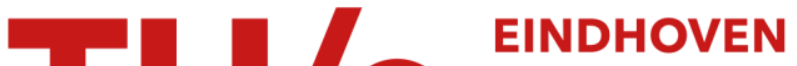

\section{Heat flux intensification by vortical flow localization in rotating convection}

Citation for published version (APA):

Kunnen, R. P. J., Clercx, H. J.H., \& Geurts, B. J. (2006). Heat flux intensification by vortical flow localization in rotating convection. Physical Review E - Statistical, Nonlinear, and Soft Matter Physics, 74(5), 056306-1/4.

[056306]. https://doi.org/10.1103/PhysRevE.74.056306

DOI:

10.1103/PhysRevE.74.056306

Document status and date:

Published: 01/01/2006

\section{Document Version:}

Publisher's PDF, also known as Version of Record (includes final page, issue and volume numbers)

\section{Please check the document version of this publication:}

- A submitted manuscript is the version of the article upon submission and before peer-review. There can be important differences between the submitted version and the official published version of record. People interested in the research are advised to contact the author for the final version of the publication, or visit the $\mathrm{DOI}$ to the publisher's website.

- The final author version and the galley proof are versions of the publication after peer review.

- The final published version features the final layout of the paper including the volume, issue and page numbers.

Link to publication

\section{General rights}

Copyright and moral rights for the publications made accessible in the public portal are retained by the authors and/or other copyright owners and it is a condition of accessing publications that users recognise and abide by the legal requirements associated with these rights.

- Users may download and print one copy of any publication from the public portal for the purpose of private study or research.

- You may not further distribute the material or use it for any profit-making activity or commercial gain

- You may freely distribute the URL identifying the publication in the public portal.

If the publication is distributed under the terms of Article 25fa of the Dutch Copyright Act, indicated by the "Taverne" license above, please follow below link for the End User Agreement:

www.tue.nl/taverne

Take down policy

If you believe that this document breaches copyright please contact us at:

openaccess@tue.nl

providing details and we will investigate your claim. 


\title{
Heat flux intensification by vortical flow localization in rotating convection
}

\author{
R. P. J. Kunnen, ${ }^{1}$ H. J. H. Clercx, ${ }^{1,2}$ and B. J. Geurts ${ }^{1,2}$ \\ ${ }^{1}$ Department of Physics, Eindhoven University of Technology, P. O. Box 513, 5600 MB Eindhoven, The Netherlands \\ ${ }^{2}$ Department of Applied Mathematics, University of Twente, P. O. Box 217, 7500 AE Enschede, The Netherlands
}

(Received 15 December 2005; revised manuscript received 19 October 2006; published 21 November 2006)

\begin{abstract}
The effect of rotation on turbulent convective flow between parallel plates has been assessed with direct numerical simulations. With increasing rotation-rate an interesting transition is observed in the vertical-velocity skewness. This transition indicates a localization of motion directed away from the wall and correlates well with changes observed in the heat flux, as well as in the thermal and viscous boundary layer thicknesses. The formation of localized intense vortical structures provides for intensified vertical heat transport through Ekman pumping. At higher rotation-rates this is counteracted by the inhibition of vertical motion by rotation as expressed in the geostrophic thermal-wind balance.
\end{abstract}

DOI: 10.1103/PhysRevE.74.056306

PACS number(s): 47.27.te, 47.32.-y

Heat transport in turbulent convection has received widespread attention in the past decades. Many studies were dedicated to determining the scaling of heat transport experimentally, e.g., [1-4], while several theoretical models were proposed to explain the scaling laws, e.g., $[2,5,6]$. However, only relatively few studies can be found that incorporate a background rotation, e.g., [1,7-11]. Rotation introduces new dynamics to the flow and has a remarkable effect on the heat transfer properties of the system. As rotation and convection coexist in many geophysical and astrophysical settings it is of importance to assess the influence of rotation on convective flow.

In this paper direct numerical simulation is used to investigate a simplified model system. A layer of fluid is rotating with a vertical axis of rotation, and is vertically constrained by walls. The horizontal directions are not bounded. A constant temperature difference between top and bottom plates is applied. Several rotation rates are used.

Three dimensionless parameters determine the flow: the Rayleigh number $\mathrm{Ra}=g \alpha \Delta T H^{3} /(\nu \kappa)$, the Taylor number $\mathrm{Ta}=\left(2 \Omega H^{2} / \nu\right)^{2}$, and the Prandtl number $\sigma=\nu / \kappa$, with $g$ the gravitational acceleration, $\alpha$ the thermal expansion coefficient, $\Delta T$ the temperature difference between bottom and top walls, $H$ the distance between the walls, $\nu$ the kinematic viscosity, $\kappa$ the thermal diffusivity, and $\Omega$ the rotation rate. The convective Rossby number $\mathrm{Ro}=\sqrt{\mathrm{Ra} /(\sigma \mathrm{Ta})}$ is used to indicate the relative importance of buoyancy and Coriolis forces, i.e., Ro $>1$ indicates that buoyancy forces are larger than Coriolis forces.

The governing equations are the Navier-Stokes and temperature equations for an incompressible rotating fluid within the Boussinesq approximation [12]:

$$
\begin{aligned}
\frac{D \mathbf{u}}{D t}+\sqrt{\frac{\sigma \mathrm{Ta}}{\mathrm{Ra}}} \hat{\mathbf{z}} \times \mathbf{u} & =-\nabla p+T \hat{\mathbf{z}}+\sqrt{\frac{\sigma}{\mathrm{Ra}}} \nabla^{2} \mathbf{u}, \\
\frac{D T}{D t} & =\frac{1}{\sqrt{\sigma \mathrm{Ra}}} \nabla^{2} T, \\
\boldsymbol{\nabla} & \cdot \mathbf{u}=0,
\end{aligned}
$$

where $\mathbf{u}$ is the velocity vector, $D / D t$ the material derivative, $\hat{\mathbf{z}}$ the unit vector in the vertical direction parallel to the axis of rotation, $p$ the reduced pressure, and $T$ the temperature. Equations (1) have been nondimensionalized with the length scale $H$, the time scale $\tau=\sqrt{H /(g \alpha \Delta T)}$, and the temperature scale $\Delta T$. The time scale $\tau$ is based on the free-fall velocity $U=\sqrt{g \alpha \Delta T H}$. These equations are solved on a rectilinear domain with periodic horizontal directions. At the bottom wall $(z=0)$ no-slip conditions for velocity and a constant temperature $T=1$ are applied. The top wall $(z=1)$ is set to a temperature $T=0$ while also applying no-slip velocity conditions. Spatial discretization is done with the symmetry-preserving finite-volume scheme as proposed in [13]. Preservation of skew symmetry of the difference operators ensures stability on any grid, while also conserving mass, momentum, and energy for inviscid flow. Time integration is done using a scheme closely related to the popular Adams-Bashforth scheme. For details on the numerical method the reader is referred to [13].

The simulations presented here were conducted at $\sigma=1$, $\mathrm{Ra}=2.5 \times 10^{6}$ and $2.5 \times 10^{7}$, comparable to simulations in [10]. The grid consists of $128^{2}$ equidistant points horizontally. In the vertical direction 64 points are placed with uneven spacing for the $\mathrm{Ra}=2.5 \times 10^{6}$ simulations, while 128 points were used for the $\mathrm{Ra}=2.5 \times 10^{7}$ simulations. Near the walls the vertical grid-point density is higher since the thin thermal and viscous boundary layers that are formed there should be adequately resolved. Values for Ta are given in Table I.

The computational domain has sides $2 \times 2 \times 1$ in the horizontal directions and the vertical direction, respectively. This domain allows for at least four characteristic convective length scales in both horizontal directions in all simulations [14]. Simulations at other horizontal domain sizes and gridpoint densities gave similar results, indicating that with these choices the flow is adequately represented.

A simulation is initialized with zero velocity and a linear vertical temperature profile with small random perturbations. A statistically stationary state was established after 50 dimensionless time units and the averaging process was started. Averaging was carried out over horizontal grid planes and in time and is denoted by $\langle\cdot\rangle$. Two quantities are of special interest for the rest of this paper, viz., the Nusselt number $\mathrm{Nu}$ and the vertical-velocity skewness $S_{w}$. The Nusselt number $\mathrm{Nu}$ is the total heat flux (convection and 
TABLE I. Simulation parameters. All simulations adopt $\sigma=1$. For some of the chosen Ta values buoyancy forces are larger than Coriolis forces $(\operatorname{Ro}>1)$, while for the others Coriolis forces are dominant over buoyancy $($ Ro $<1)$.

\begin{tabular}{cccc}
\hline \hline \multicolumn{2}{c}{$\mathrm{Ra}=2.5 \times 10^{6}$} & \multicolumn{2}{c}{$\mathrm{Ra}=2.5 \times 10^{7}$} \\
$\mathrm{Ta}$ & $\mathrm{Ro}$ & $\mathrm{Ta}$ & $\mathrm{Ro}$ \\
\hline 0 & $\infty$ & & \\
$1.6 \times 10^{5}$ & 4.00 & $1.6 \times 10^{6}$ & 4.00 \\
$2.4 \times 10^{5}$ & 3.20 & $2.4 \times 10^{6}$ & 3.20 \\
$4.0 \times 10^{5}$ & 2.50 & $4.0 \times 10^{6}$ & 2.50 \\
$1.4 \times 10^{6}$ & 1.33 & $1.4 \times 10^{7}$ & 1.33 \\
$4.5 \times 10^{6}$ & 0.75 & $2.5 \times 10^{7}$ & 1.00 \\
$1.0 \times 10^{7}$ & 0.50 & $4.5 \times 10^{7}$ & 0.75 \\
$1.6 \times 10^{7}$ & 0.40 & $1.0 \times 10^{8}$ & 0.50 \\
$2.3 \times 10^{7}$ & 0.33 & $1.6 \times 10^{8}$ & 0.40 \\
$4.0 \times 10^{7 \mathrm{a}}$ & 0.25 & $2.3 \times 10^{8}$ & 0.33 \\
$1.0 \times 10^{8 \mathrm{a}}$ & 0.16 & & \\
\hline \hline
\end{tabular}

$\overline{\mathrm{a}}$ These simulations also used the $128^{3}$ grid in order to resolve the thin viscous boundary layer.

conduction) normalized by the conductive heat flux that would be present in absence of convection. Nu can be obtained from the wall-normal derivative of the average temperature [15]: $\mathrm{Nu}=\partial\langle T\rangle /\left.\partial z\right|_{\text {wall }}$. The vertical-velocity skewness $S_{w}$ is defined as $S_{w}=\left\langle w^{3}\right\rangle /\left\langle w^{2}\right\rangle^{3 / 2}$. The averaging for the Nusselt number converged quite rapidly; within 20 dimensionless time units the value was within $1 \%$ of its final value. For the vertical-velocity skewness $S_{w}$, however, a longer time of averaging was necessary. In order to satisfactorily retain the symmetry with respect to the horizontal midplane an averaging of 300 time units was found necessary.

An interpretation of $S_{w}$, as given in [16], concerns the spatial distribution of upward and downward motions in horizontal cross sections of the domain. If the area occupied by patches of upward motion is larger than that occupied by downward motion the average downward velocity has to be larger than the average upward velocity as the flow is incompressible. The resulting vertical-velocity skewness will then be negative. Similarly, a large positive skewness indicates strongly localized upward motion. Hence the verticalvelocity skewness is indicative of the structuring of convective flows. Additionally, $S_{w}$ is a quantity that is used in theories of turbulent diffusion in the planetary boundary layer (see, e.g., [17]), and its value is thus an interesting property in itself.

Figure 1 shows the vertical-velocity skewness as a function of the vertical coordinate and Rossby number for $\mathrm{Ra}=2.5 \times 10^{6}$. Only the bottom half of the vertical extent is shown because of symmetry. The S-shaped curve for the nonrotating case $(\mathrm{Ro}=\infty, \times)$ is shown for the full vertical extent in the inset; it is very similar to the profiles found in previous numerical simulations $[15,16]$. In these references, the shape is explained by noting that close to the walls only a few strong plumes coming from the other side can penetrate. Thereby the motion of cold (hot) fluid towards the hot (cold) wall is becoming more and more localized when

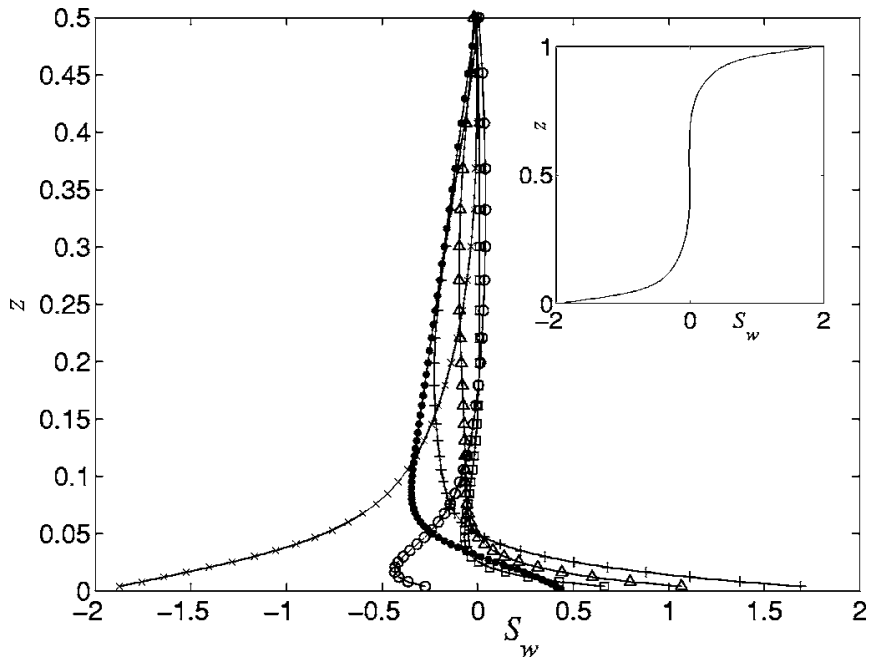

FIG. 1. Skewness of vertical velocity for $\mathrm{Ra}=2.5 \times 10^{6}$. For clarity only six curves are included: $R o=\infty(\times), 4.00(\bigcirc), 2.50(\square)$, $1.33(\triangle), 0.75(+)$, and $0.16(\bullet)$. Only the lower half of the vertical extent is shown given the symmetry of top and bottom halves. In the inset the full vertical extent of $S_{w}$ is depicted for the case Ro $=\infty$.

approaching the wall, and thus increasing the absolute value of $S_{w}$.

Interestingly, with decreasing Ro the near-wall tails of the curves gradually change sign, pointing to a change in flow structuring near the walls. At Ro $=\infty$ near the walls the motion toward the wall is concentrated in localized intensive structures, i.e., $S_{w}<0$ near the bottom wall and $S_{w}>0$ near the top. A gradual transition can be observed with decreasing Ro toward a final situation where the motion directed away from the walls is dominated by localized intense structures, resulting in a positive $S_{w}$ near the bottom wall and $S_{w}$ negative near the top wall. The extreme case is around Ro=0.75; for lower Ro the near-wall $S_{w}$ decreases again (see, e.g., the curve for Ro=0.16 in Fig. 1).

The near-wall transition of $S_{w}$ was also found in simulations with $\sigma=0.25$ and 4 , at the same Rossby $(\operatorname{Ro}=0.75)$ and Rayleigh numbers $\left(\mathrm{Ra}=2.5 \times 10^{6}\right)$. In the simulation at $\mathrm{Ra}=2.5 \times 10^{7}, \mathrm{Ro}=0.75$, the same effect was found, with $S_{w}$ being even closer to zero in most of the domain, while growing in narrow regions close to the bottom and top walls. This case was very similar to the $S_{w}$ curve presented in [10], albeit at lower Ra.

Summarizing, at low (and zero) Taylor numbers the flow toward the boundaries is localized in smaller structures with a negative (positive) $S_{w}$ near the bottom (top) wall, while at larger Taylor numbers $\left(\mathrm{Ta} \gtrsim 4 \times 10^{5}\right.$ at $\left.\mathrm{Ra}=2.5 \times 10^{6}\right)$ the flow directed away from the boundaries is concentrated in localized plumes with a positive (negative) $S_{w}$ near the bottom (top) wall.

Having established that the skewness of vertical velocity is indicative of convective flow structuring, the next step is an inspection of the actual structures that are found in the flow. Following [16], horizontal cross-sections of vertical velocity close to the bottom wall $(z=0.015)$ are presented in Figs. 2(a) and 2(b) at Ro= $\infty$ and 0.75 , respectively.

A striking observation is that under rotation there are 


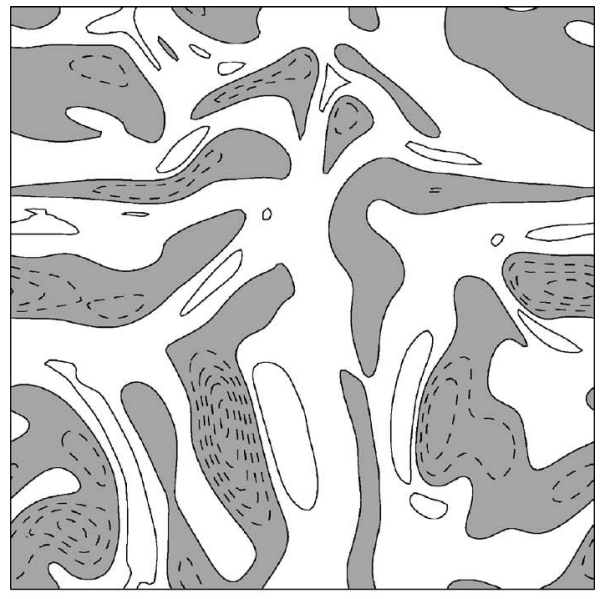

(a)

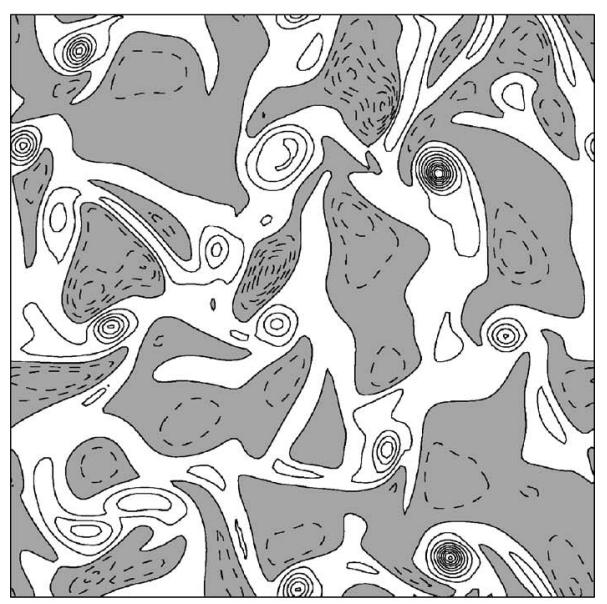

(b)

FIG. 2. Horizontal cross sections of snapshots of vertical velocity. The solid contours on the white areas indicate upward motion, while the dashed contours and the gray areas are for downward motion. The contour increment is 0.01 . The cross sections are taken at height $z=0.015$, inside the viscous boundary layer near the bottom wall. $\mathrm{Ro}=(\mathrm{a}) \infty$ and (b) 0.75 .

many small structures with a large upward velocity. These are found to be so-called thermal vortices. As fluid converges to a plume-formation site it is spun up cyclonically due to angular momentum conservation, and the resulting plume therefore contains cyclonic (positive) vorticity. Note that this is true for plumes emanating from both top and bottom walls. With increasing Ta (decreasing Ro) the number of these thermal vortices is found to be increasing, or, equivalently, their separation is decreasing. This was also observed in laboratory experiments $[14,18]$ and numerical simulations [10].

The occurrence of vortical structures near solid boundaries reveals an additional mechanism for vertical mass and heat transport, Ekman pumping. A vortical flow of positive vertical vorticity is matched to the no-slip wall via an Ekman boundary layer, as described in, e.g., [19]. The Ekman layer, unlike the regular viscous Prandtl boundary layer, induces a vertical motion outside its confines. Transport of fluid away from the walls is concentrated in localized vortices with intense vertical transport induced by Ekman pumping. How-

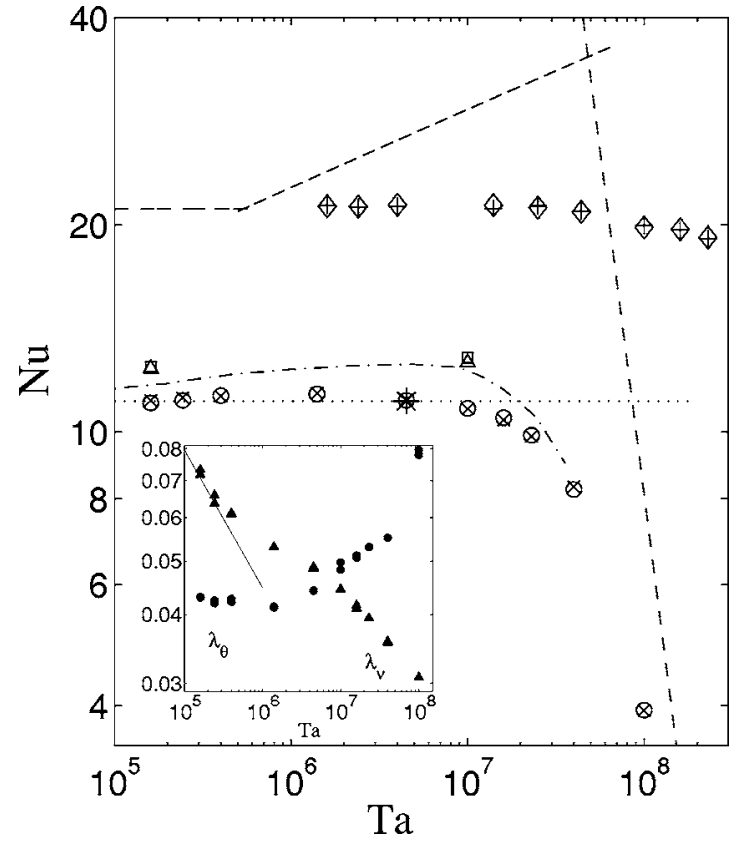

FIG. 3. Dependence of Nu on Ta. For $\mathrm{Ra}=2.5 \times 10^{6}$ the crosses denote $\mathrm{Nu}$ calculated at the top wall, the circles are for $\mathrm{Nu}$ calculated at the bottom wall, and the value for $\mathrm{Ta}=0$ is indicated by the dotted line. The pluses and diamonds give $\mathrm{Nu}$ at top and bottom walls for $\mathrm{Ra}=2.5 \times 10^{7}$, respectively. Rossby's experimental result [1] for water $\left(\sigma=6.8, \mathrm{Ra}=2.5 \times 10^{6}\right)$ is depicted with the dashdotted line. The dashed lines are upper bounds for the heat flux for $\sigma \rightarrow \infty, \mathrm{Ra}=2.5 \times 10^{6}$ obtained with a variational method in [22]. The inset shows the dependence of the viscous boundary layer thickness $\lambda_{\nu}$ (filled triangles) and the thermal boundary layer thickness $\lambda_{\theta}$ (filled circles) on Ta. The solid line is the classical Ekman boundary layer thickness.

ever, as a hot (cold) plume rises (sinks) and approaches the other wall its vorticity decreases and can even become negative (anticyclonic) by vortex tube compression when the fluid is spread radially outward. The ensemble of these effects is that near the walls small intense cyclonic vortices with intense vertical transport are scattered between much larger regions of radially spreading plumes coming from the other side. Localized and intense Ekman pumping and the radial spread of plumes when approaching the other side are the mechanisms that are responsible for the transition of $S_{w}$ near the walls under rotation.

The local changes in flow structuring under rotation also have an effect on the heat transfer properties of the entire domain. In Fig. 3 the Nusselt number $\mathrm{Nu}$ is shown as a function of the Taylor number Ta for the two Ra values considered. For $\mathrm{Ra}=2.5 \times 10^{6}$, at moderate values $0<\mathrm{Ta} \leqq 5 \times 10^{6}$ the heat flux is higher than in the nonrotating situation $(\mathrm{Nu}$ increasing under rotation was also noticed in $[1,7,11]$ ), coincident with the transition of $S_{w}$ near the walls. In this regime the heat flux can increase since $S_{w}$ indicates an increasing localization of velocity directed away from the boundary that, along with the cyclonic nature of these structures, gives rise to localized strong Ekman pumping as an efficient mechanism for extracting heat from the boundary layer. The $\mathrm{Ra}=2.5 \times 10^{7}$ series shows a quantitatively similar $\mathrm{Nu}$ increase. 
At higher Ta the extremal $S_{w}$ values are reduced (cf. Fig. 1). In this regime, with increasing Ta, the convective motions become more and more suppressed. This is a consequence of rotation on the convective flow: under geostrophic conditions (steady flow, inertial and viscous forces negligible compared to the Coriolis force, i.e., small Ro and large Ta) the bulk flow is in the so-called thermal-wind balance [19] and vertical motion is independent of the vertical coordinate. In combination with the no-slip boundaries it can be stated that the vertical motion tends to zero. Hence, rotation suppresses vertical motion, and thus also convection, in the geostrophic bulk regime. This explains the rapid decrease of $\mathrm{Nu}$ in the high-Ta regime.

For comparison, other numerical and experimental results for $\mathrm{Nu}$ are included in Fig. 3. The dash-dotted line gives the experimental results from [1] using water $(\sigma=6.8)$. As a further comparison, two simulations at this $\sigma$ have been carried out; these values are represented by squares and triangles for bottom and top walls, respectively. There is a minor gap between the experimental results and our simulations. This can be explained by the finite flow domain in the experiment. The sidewalls cause the measured $\mathrm{Nu}$ values to be lower than in the horizontally unbounded case. This flow-domain effect is also depicted in [15], where $\mathrm{Nu}$ obtained from horizontally unbounded simulations is compared to finite-domain experimental results from [20], both using a $\sigma=0.7$ fluid. The value taken from the double-periodic simulations in [10] $(\sigma=1)$, indicated by the star in Fig. 3, matches very well to the current study.

There is also a wealth of studies on upper bounds for $\mathrm{Nu}$ to be found in the literature. A rigorous analytic bound for $\sigma \rightarrow \infty$ was derived in [21]. However, in the current parameter regime this bound is about a factor 20 above the simulation results. A bound, also for $\sigma \rightarrow \infty$, that was found to be better suited here was obtained by a variational method in [22]; it is depicted with the dashed lines in Fig. 3. The bound consists of three separate regimes, and even the increase of
$\mathrm{Nu}$ at moderate $\mathrm{Ta}$ is included. Also the rapid decay of $\mathrm{Nu}$ for high Ta is clearly represented. The discrepancy in the actual values can be explained by the differences in $\sigma$. For values up to $\sigma \approx 6$ the heat transfer is larger for larger- $\sigma$ fluids. When $\sigma$ grows larger than $6, \mathrm{Nu}$ will be dropping again, albeit only very slightly $[23,24]$.

In the inset in Fig. 3 the dependence of the viscous boundary layer thickness $\lambda_{\nu}$ and the thermal boundary layer thickness $\lambda_{\theta}$ on Ta is shown. The boundary layer thicknesses were defined as the heights at which the horizontal rms velocities and the rms temperature values are maximal. It can be seen that $\lambda_{\theta}$ is roughly inversely proportional to $\mathrm{Nu}$, as expected. An interesting transition was found for $\lambda_{\nu}$, right around the Ta value of maximum heat flux. The transition is from the (nondimensionalized) classical Ekman boundary layer thickness $\sqrt{\nu /\left(\Omega H^{2}\right)}=\sqrt{2} \mathrm{Ta}^{-1 / 4}$ (indicated with the solid line) to a flatter profile. Coarse-gridded simulations at lower Ta values have revealed that $\lambda_{\nu}$ does not increase by much more; it instead approaches the nonrotating $\lambda_{\nu}$ value which is just slightly below the value at $\mathrm{Ta}=1.6 \times 10^{5}$.

We have used the skewness of vertical velocity as a measure for localization of vertical motion to clarify the heat transfer of rotating convection at different rotation rates. As the rotation rate increases the motion directed away from the boundaries becomes more and more localized in slender vortical plumes with cyclonic vorticity. Ekman pumping in these vortices increases extraction of heat from the boundary layers and thus also the heat flux. At even higher rotation rates the heat flux decreases as the quasigeostrophic conditions in the bulk increasingly inhibit convection.

R.P.J.K. wishes to thank the Foundation for Fundamental Research of Matter (FOM) for financial support. This work was sponsored by the National Computing Facilities Foundation (NCF) for the use of supercomputer facilities, with financial support from the Netherlands Organisation for Scientific Research (NWO).
[1] H. T. Rossby, J. Fluid Mech. 36, 309 (1969).

[2] B. Castaing et al., J. Fluid Mech. 204, 1 (1989).

[3] J. J. Niemela et al., Nature (London) 404, 837 (2000).

[4] E. Brown et al., Phys. Fluids 17, 075108 (2005).

[5] B. I. Shraiman and E. D. Siggia, Phys. Rev. A 42, 3650 (1990).

[6] S. Grossmann and D. Lohse, J. Fluid Mech. 407, 27 (2000).

[7] J. M. Pfotenhauer, P. G. J. Lucas, and R. J. Donnelly, J. Fluid Mech. 145, 239 (1984).

[8] F. Zhong, R. E. Ecke, and V. Steinberg, J. Fluid Mech. 249, 135 (1993).

[9] A. P. Bassom and K. Zhang, Geophys. Astrophys. Fluid Dyn. 76, 223 (1994).

[10] K. Julien, S. Legg, J. McWilliams, and J. Werne, J. Fluid Mech. 322, 243 (1996).

[11] Y. Liu and R. E. Ecke, Phys. Rev. Lett. 79, 2257 (1997).

[12] S. Chandrasekhar, Hydrodynamic and Hydromagnetic Stability (Oxford University Press, Oxford, 1961).
[13] R. W. C. P. Verstappen and A. E. P. Veldman, J. Comput. Phys. 187, 343 (2003).

[14] S. Sakai, J. Fluid Mech. 333, 85 (1997).

[15] R. M. Kerr, J. Fluid Mech. 310, 139 (1996).

[16] C.-H. Moeng and R. Rotunno, J. Atmos. Sci. 47, 1149 (1990).

[17] J. W. Deardorff and G. E. Willis, Boundary-Layer Meteorol. 32, 205 (1985).

[18] B. M. Boubnov and G. S. Golitsyn, J. Fluid Mech. 167, 503 (1986).

[19] A. E. Gill, Atmosphere-Ocean Dynamics (Academic Press, New York, 1982).

[20] X.-Z. Wu and A. Libchaber, Phys. Rev. A 45, 842 (1992).

[21] P. Constantin, C. Hallstrom, and V. Putkaradze, Physica D 125, 275 (1999).

[22] C. Hunter and N. Riahi, J. Fluid Mech. 72, 433 (1975).

[23] R. M. Kerr and J. R. Herring, J. Fluid Mech. 419, 325 (2000).

[24] M. Sprague, K. Julien, E. Knobloch, and J. Werne, J. Fluid Mech. 551, 141 (2006). 